\title{
Ciclo biológico de Argas miniatus em laboratório: estágio adulto
}

\author{
Life cycle of Argas (Persicargas) miniatus in the laboratory: adult stage
}

\author{
Huarrisson Azevedo Santos $^{I}$ Isabele da Costa Angelo ${ }^{I}$ Marcos Pinheiro Franque \\ Cristiane Divan BaldaniI Sandra Maria Gomes Thomé ${ }^{I I I}$ João Luiz Horacio Faccini ${ }^{\mathrm{I}}$ \\ Carlos Luiz Massard
}

\section{RESUMO}

\begin{abstract}
Os parâmetros do ciclo biológico de Argas (Persicargas) miniatus, fase adulta, foram estudados em condições controladas $\left(27 \pm 1^{\circ} \mathrm{C}\right.$ e $80 \pm 10 \%$ UR) e em condições ambientais não controladas (ambiente de laboratório nas estações chuvosa e seca). Nessas condições, os machos se originaram preferencialmente de N2 e as fêmeas de N3. As fêmeas realizaram até 18 posturas (em condições controladas), enquanto que as fêmeas mantidas nas estações chuvosa e seca realizaram até 9 e 12 posturas, respectivamente. O número total de ovos foi 1350 (em condições controladas), 443 ovos na estação chuvosa e 894 ovos na estação seca. Em jejum, os carrapatos machos sobreviveram por 165 dias (condições controladas) e 135 dias em ambiente de laboratório; as fêmeas sobreviveram por 300 dias em condições controladas e 240 dias no ambiente de laboratório. Nas condições ambientais não controladas, os parâmetros biológicos do estágio adulto de A. (P.) miniatus sofreram alterações negativas, principalmente durante a estação chuvosa.
\end{abstract}

Palavras-chave: ciclo biológico, Argas (Persicargas) miniatus, estágio adulto, Gallus gallus.

\section{ABSTRACT}

The life cycle of Argas (Persicargas) miniatus in adult stage was studied in controlled conditions $\left(27 \pm 1^{\circ} \mathrm{C}\right.$ and $80 \pm 10 \%$ RH) and uncontrolled temperature and humidity conditions (room conditions during wet and dry season). Males originated preferentially from $\mathrm{N} 2$ and females from N3. Females in controlled conditions laid eggs up to 18 times, while females in uncontrolled room conditions, during wet and dry seasons, laid eggs 9 to 12 times, respectively. In controlled conditions, females laid 1350 eggs, whereas in uncontrolled conditions females laid 443 eggs during the wet season and 894 eggs during the dry season. Male ticks survived without feeding for 165 days in controlled conditions and 135 days in uncontrolled conditions, while females survived for 300 days in controlled conditions and 240 days in uncontrolled conditions. In ambient conditions, biological parameters of $\boldsymbol{A}$. (P.) miniatus adult stage were negatively affected especially during the wet season when compared with adult stage kept in controlled condition.

Key words: life cycle, Argas (Persicargas) miniatus, adult stage, Gallus gallus.

\section{INTRODUÇÃO}

Argas (Persicargas) miniatus Koch, 1844, é uma espécie de carrapato neotropical encontrada na América do Norte, Central e principalmente na América do Sul. Esse argasídeo se mantém na natureza principalmente em pequenas criações domésticas de Gallus gallus, nas quais pode determinar perdas na produtividade, decorrentes do hematofagismo, da transmissão de agentes patogênicos, como Borrelia anserina (MARCHOUX \& SALIMBENI, 1903) e da paralisia induzida pelas larvas em aves jovens (MAGALHÃES et al., 1987).

É um carrapato heteroxeno e com hábito alimentar noturno. Durante o processo de alimentação, a larva permanece sobre o hospedeiro durante dias, enquanto ninfas e adultos realizam seus repastos sanguíneos em poucos minutos. Na fase de vida livre, são encontrados em abrigos e ninhos de seus

'Departamento de Parasitologia Animal, Universidade Federal Rural do Rio de Janeiro (UFRRJ), 23890-000, Seropédica, RJ, Brasil. E-mail: huarrisson@yahoo.com.br. Autor para correspondência.

"Departamento de Medicina e Cirurgia Veterinária, UFRRJ, Seropédica, RJ, Brasil.

IIIDepartamento de Epidemiologia e Saúde Pública da UFRRJ, Seropédica, RJ, Brasil. 
hospedeiros, onde ocorre a muda e a cópula (ROHR, 1909). Possui de dois a quatro instares ninfais (MAGALHÃES, 1979) e os adultos se desenvolvem a partir do segundo instar ninfal. As fêmeas dependem do repasto sanguíneo e de pelo menos uma cópula inicial para realização da postura. Elas podem realizar até 16 posturas durante toda a duração do ciclo biológico, com média de 136 ovos postura ${ }^{-1}$ (RHOR, 1909). Com uma única cópula, uma fêmea realiza até seis posturas intercaladas com o repasto sanguíneo (RHOR, 1909; MAGALHÃES, 1979). Os ovos passam por um período de incubação que varia de 12 a 41 dias, dependendo da temperatura (RHOR, 1909) e o percentual médio de eclosão é de $82,7 \%$ (SCHUMAKER \& OBA, 1988). Em condições de temperatura e UR controladas, o ciclo completo pode durar até 201 dias, ao passo que, em condições naturais, pode chegar até 317 dias (SCHUMAKER \& OBA, 1988).

Estudos sobre o ciclo biológico de $\boldsymbol{A}$. (P.) miniatus foram realizados por ROHR (1909), MAGALHÃES (1979), SCHUMAKER \& OBA(1988)e SANTOS et al. (2008). No entanto, alguns aspectos do ciclo biológico do estágio adulto ainda não foram descritos, tais como: longevidade, reprodução estimada, índice nutricional, número de ovos por grama de postura, influência do peso sobre a razão sexual, entre outros. Este trabalho descreve essas etapas do ciclo biológico do estágio adulto de $\boldsymbol{A}$. (P.) miniatus em condições controladas $\left(27 \pm 1^{\circ} \mathrm{C}\right.$ e $80 \pm 10 \%$ (UR)) e em condições ambientais não controladas, contribuindo, desse modo, para melhorar o conhecimento sobre o ciclo biológico dessa espécie.

\section{MATERIAL E MÉTODOS}

Obtenção e manutenção da colônia

Fêmeas ingurgitadas de $\boldsymbol{A}$. (P.) miniatus foram obtidas de uma pequena criação de galinhas domésticas (G gallus), localizada em Brasília, DF. Os ovos produzidos pelas fêmeas foram separados, acondicionados em seringas plásticas descartáveis adaptadas para o uso e incubados a $27 \pm 1^{\circ} \mathrm{C}$ e $80 \pm 10 \%$ de UR. As larvas eclodidas foram mantidas nessa mesma condição, assim como os demais estágios. $\mathrm{O}$ estágio larval foi alimentado em aves com idade inferior a uma semana, conforme SANTOS et al. (2008). A alimentação dos estágios ninfais procedeu-se conforme descrito por KAISER (1965). A partir dessa colônia, foram obtidos os estágios adultos utilizados neste trabalho.

Delineamento experimental

Os carrapatos adultos foram alimentados individualmente em aves (adultos de G. gallus) adquiridas em estabelecimento comercial. Após a alimentação, uma parte dos carrapatos foi mantida em estufa climatizada do tipo B.O.D. (Biochemical Oxygen Demand) a $27 \pm 1^{\circ} \mathrm{C}, 80 \pm 10 \%$ de UR e escotofase e a outra em ambiente de laboratório. Nesta última condição, os dados foram obtidos durante as estações seca (ES) (maio a outubro) e chuvosa (ECH) (novembro a abril) dos anos de 2004, 2005 e 2006, com temperatura média variando entre $16,7^{\circ} \mathrm{C}$ e $34,4^{\circ} \mathrm{C}$ e UR média variando entre $51,3 \%$ e $87,6 \%$, respectivamente.

\section{Avaliações biológicas}

Foram avaliados: período médio de alimentação (número de dias entre a fixação e o desprendimento natural), peso médio da fêmea antes da alimentação, peso médio da fêmea no início da postura, peso médio da fêmea no final da postura, peso médio do macho antes da alimentação, peso médio do macho depois da alimentação, percentual de mortalidade (razão entre o número de carrapatos mortos entre uma alimentação e outra e o número total de carrapatos, expresso em percentagem), massa média de sangue ingerido (diferença entre o peso dos carrapatos após a alimentação e liberação do líquido coxal sobre o peso antes da alimentação), período médio de pré-postura (número de dias entre o início da alimentação da fêmea e o início da postura), período médio de postura (número de dias entre o início e o fim da postura), número médio de ovos por grama de postura (LABRUNA et al., 1997), massa média de ovos diária (massa média dos ovos produzidos a cada 24 horas), massa média de ovos por postura, massa média de ovos total (massa média dos ovos produzidos em todas as posturas); período médio de incubação dos ovos (número de dias entre o início da postura e o início da eclosão larval), percentual médio de eclosão larval (obtido por avaliação visual da quantidade de larvas eclodidas em relação à massa total de ovos), reprodução estimada (RE) (DRUMMOND et al., 1971), índice nutricional (IN) (BENNETT, 1974) e número médio de posturas (número de vezes que uma fêmea realizou postura após a alimentação e cópula).

Para estudar a longevidade, trinta machos e trinta fêmeas foram mantidos individualmente em seringas plásticas adaptadas para essa finalidade e acondicionados em B.O.D $\left(27 \pm 1^{\circ} \mathrm{C}\right.$ e $80 \pm 10 \%$ de UR $)$ ou no ambiente de laboratório. A cada 15 dias, todos os carrapatos foram examinados até que fosse constatada a morte de todos os exemplares, conforme SANTOS et al. (2008).

Análise estatística

Os dados que apresentaram normalidade foram submetidos à análise de variância e as médias 
comparadas pelo teste $t$-Student, com $5 \%$ de significância. Os dados não paramétricos foram submetidos ao teste de Kruskal-Wallis e as médias comparadas pelo teste de Student-Neumann-Keuls, com $5 \%$ de significância. Os dados que apresentaram normalidade foram avaliados conjuntamente, a fim de verificar a tendência e o grau de associação pelo método de Pearson com 5\% de probabilidade e os que não apresentaram normalidade foram submetidos ao método de Sperman ( $5 \%$ de probabilidade).

\section{RESULTADOS E DISCUSSÃO}

Proporção macho:fêmea

Os carrapatos adultos mantidos nas três condições experimentais se desenvolveram a partir de ninfas de segundo (N2) e terceiro instar (N3). A proporção macho/fêmea de adultos que se desenvolveram a partir de $\mathrm{N} 2$ foi de 1,5:1 (35 ठౌe 20 q) em B.O.D; 2:1 (28ðౌe 14우) na ECH e 1,88:1 (32б'e 17 우) na $\mathrm{ES}(\mathrm{P}<0,05)$. Em relação à proporção macho/fêmea de adultos originados de $\mathrm{N} 3$, foi observada relação 1:1,74 (43 ठౌe 75 q) em B.O.D; 1:1,68 (34 ठ'e 57 q) na ECH e $1: 1,74$ (38 đ’e 66 o $)$ na ES $(P<0,05)$. Em cada condição experimental, houve uma proporção significativamente maior $(\mathrm{p}<0,05)$ de machos originados de $\mathrm{N} 2 \mathrm{e}$ de fêmeas originadas de $\mathrm{N} 3$.

KAISER (1965) atribuiu o fato de fêmeas se originarem de $\mathrm{N} 3$ devido a uma maior necessidade nutricional para maturação sexual, bem como pelo fato de a fêmea ser maior e possuir um sistema reprodutivo mais complexo. Segundo KHALIL (1979), a proporção entre machos:fêmeas de $\boldsymbol{A}$. (P.) persicus é 1: 1,1, em que a maioria dos machos ( $81 \%$ ) originaram-se de $\mathrm{N} 2 \mathrm{e}$ $19 \%$ de N3. Em relação às fêmeas, $31 \%$ se originaram de N2, $45 \%$ de N3 e $25 \%$ de N4. Em linhas gerais, esse resultado é similar ao obtido em nosso experimento.

\section{Tempo de alimentação}

As fêmeas se alimentaram por um período de 7-46 minutos em todas as condições estudadas: 17,13 $\pm 7,92$ minutos em B.O.D, 18,25 $\pm 5,30$ minutos na $\mathrm{ECH}$ e $17,80 \pm 6,39$ minutos na ES, não existindo diferença significativa $(\mathrm{P}>0,05)$ (Tabela 1). Em $\boldsymbol{A}$. (P.) arboreus, o período de alimentação de adultos não se alterou quando mantidos em diferentes temperaturas $\mathrm{e}$ umidades antes da alimentação (HAFEZ et al., 1972). O período médio de alimentação de machos em B.O.D, ECH e ES foi de 13,96 $\pm 4,83$ minutos, $14,36 \pm 4,75$ minutos

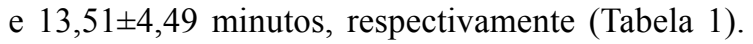
Segundo HAFEZ et al. (1972), o período de alimentação de $\boldsymbol{A}$. (P.) arboreus variou entre 9 e 180 minutos, com média de 46,8 minutos na primeira alimentação e 53,9 minutos nas alimentações seguintes. Neste estudo, foi verificado que machos de $\boldsymbol{A}$. (P.) miniatus se alimentaram por um período significativamente $(\mathrm{P}<0,05)$ menor, corroborando estudos realizados por ROHR (1909) e SCHUMAKER \& OBA (1988). Um período de alimentação mais longo foi descrito para machos de $\boldsymbol{A}$. (P.) robertsi (HOOGSTRAAL et al., 1975), diferença, provavelmente, devido às características relacionadas ao peso e à necessidade fisiológica.

Peso médio

O peso médio dos machos antes e após a alimentação foi inferior $(\mathrm{P}<0,05)$ ao das fêmeas nas três condições estudadas. $\mathrm{O}$ peso médio das fêmeas antes dos repastos sanguíneos mantidas em B.O.D $(14,87 \pm 2,46 \mathrm{mg})$ e no ambiente durante a ES $(13,01 \pm 2,61 \mathrm{mg})$ não variou $(\mathrm{P}>0,05)$, porém diferiu $(\mathrm{p}<0,05)$ do peso das fêmeas na $\mathrm{ECH}(14,73 \pm 2,90 \mathrm{mg})$. $\mathrm{O}$ clima durante a estação seca no Município de Seropédica, RJ, é caracterizado por possuir temperaturas entre $16^{\circ} \mathrm{C} \mathrm{e} 27^{\circ} \mathrm{C}$ e UR $45 \%$ a $70 \%$, o que possivelmente contribuiu para que o peso do carrapato fosse menor. Segundo SONENSHINE (1993), em condições de baixa umidade, os carrapatos perdem água através da cutícula pela transpiração, sofrendo assim dessecação e perda de peso. O peso médio dos machos antes e após o ingurgitamento foi de 7,97 $\pm 1,52 \mathrm{mg}$ e $15,03 \pm 2,83 \mathrm{mg}$, quando mantidos em B.O.D, 7,20 $\pm 1,40 \mathrm{mg}$ e $14,01 \pm 2,88 \mathrm{mg}$ nas ES e $7,98 \pm 1,67 \mathrm{mg}$ e $15,46 \pm 2,52 \mathrm{mg}$ nas ECH, respectivamente (Tabela 1).

Massa de sangue ingerido

Durante a alimentação, cada fêmea ingeriu

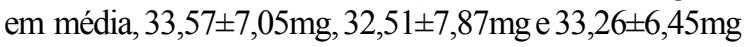
de sangue em B.O.D e nas ECH e ES, respectivamente, sem diferença entre elas $(\mathrm{P}>0,05)$. Após o repasto sanguíneo, as fêmeas de $\boldsymbol{A}$. (P.) miniatus alcançaram até 3,34 vezes o valor de seu peso antes da alimentação, enquanto que os machos somente 1,95 vezes. SCHUMAKER \& OBA (1988) observaram que fêmeas e machos aumentam o seu peso após o repasto sanguíneo em aproximadamente duas vezes, com ligeira superioridade para as fêmeas.

Pré-postura, postura e incubação

Os períodos de pré-postura, postura e incubação estão descritos na tabela 1. Não houve diferença significativa entre as condições estudadas, porém esses períodos se prolongaram na ES, provavelmente devido à baixa temperatura, que diminuiu o metabolismo das fêmeas. EL KAMMAH \& WAHAB (1979) verificaram para $\boldsymbol{A}$. (P.) persicus um período de pré-postura de $7,9 \pm 2,1$ dias em condições 
Tabela 1 - Valores médios e desvio padrão dos aspectos biológicos da fase não parasitária de fêmeas de Argas (Persicargas) miniatus mantidas em estufa climatizada do tipo B.O.D a $27 \pm 1,0^{\circ} \mathrm{C}$ e $80 \pm 10 \%$ de umidade relativa (UR) e em condição ambiental durante as estações secas e chuvosas dos anos de 2004, 2005 e 2006.

\begin{tabular}{lccc}
\hline & & & \\
Aspectos biológicos & BOD $\left(27 \pm 1,0^{\circ} \mathrm{C}\right.$ e $80 \pm 10 \%$ UR) & Estação chuvosa & Estação seca \\
\hline PMFAA (mg) & $14,87 \pm 2,46^{\mathrm{b}}$ & $14,73 \pm 2,90^{\mathrm{b}}$ & $13,01 \pm 2,61^{\mathrm{a}}$ \\
PMIP (mg) & $46,89 \pm 9,44^{\mathrm{a}}$ & $46,43 \pm 10,37^{\mathrm{ab}}$ & $49,00 \pm 9,19^{\mathrm{a}}$ \\
PMFP (mg) & $15,29 \pm 2,61^{\mathrm{a}}$ & $13,92 \pm 2,78^{\mathrm{b}}$ & $15,75 \pm 2,93^{\mathrm{a}}$ \\
PMMAA (mg) & $7,97 \pm 1,52^{\mathrm{a}}$ & $7,20 \pm 1,40^{\mathrm{b}}$ & $7,98 \pm 1,67^{\mathrm{a}}$ \\
PMMDA (mg) & $15,03 \pm 2,83^{\mathrm{a}}$ & $14,02 \pm 2,88^{\mathrm{b}}$ & $15,46 \pm 2,52^{\mathrm{a}}$ \\
MMSI (mg) & $33,57 \pm 7,05^{\mathrm{a}}$ & $32,51 \pm 7,87^{\mathrm{a}}$ & $33,26 \pm 6,45^{\mathrm{a}}$ \\
PMPP (dias) & $4,00 \pm 0,90^{\mathrm{c}}$ & $5,16 \pm 1,02^{\mathrm{b}}$ & $5,79 \pm 0,88^{\mathrm{a}}$ \\
PMP (dias) & $8,46 \pm 1,1^{\mathrm{b}}$ & $6,87 \pm 1,11^{\mathrm{c}}$ & $9,69 \pm 1,32^{\mathrm{a}}$ \\
NMO (unidade) & $100,14 \pm 22,21^{\mathrm{b}}$ & $81,95 \pm 15,2^{\mathrm{c}}$ & $109,70 \pm 9,64^{\mathrm{a}}$ \\
MMO (mg) & $13,70 \pm 3,04^{\mathrm{b}}$ & $11,21 \pm 2,0^{\mathrm{c}}$ & $15,01 \pm 1,32^{\mathrm{a}}$ \\
PMI (dias) & $9,14 \pm 1,0^{\mathrm{c}}$ & $12,56 \pm 1,4^{\mathrm{b}}$ & $14,50 \pm 1,1^{\mathrm{a}}$ \\
PMEL (\%) & $90,59 \pm 6,53^{\mathrm{a}}$ & $85,57 \pm 6,42^{\mathrm{b}}$ & $86,08 \pm 5,9^{\mathrm{b}}$ \\
RE (\%) & $38,26 \pm 6,74^{\mathrm{a}}$ & $31,44 \pm 6,36^{\mathrm{b}}$ & $40,28 \pm 6,71^{\mathrm{a}}$ \\
IN (\%) & $41,86 \pm 6,5^{\mathrm{b}}$ & $36,68 \pm 6,8^{\mathrm{c}}$ & $47,17 \pm 6,73^{\mathrm{a}}$ \\
NMP(unidade) & $13,48 \pm 5,74^{\mathrm{c}}$ & $5,41 \pm 2,65^{\mathrm{a}}$ & $8,15 \pm 3,16^{\mathrm{b}}$ \\
\hline
\end{tabular}

${ }^{a}$ Valores nas linhas, seguidos de mesma letra não diferem entre si pelo teste Kruskal-Wallis com $\mathrm{P}<0,05$. PMFAA: peso médio da fêmea antes da alimentação; PMIP: peso médio da fêmea no início da postura; PMFP: peso médio da fêmea no final da postura; PMMAA: peso médio do macho antes da alimentação; PMMDA: peso médio do macho depois da alimentação; MMSI: massa média de sangue ingerido; PMPP: período médio pré-postura; PMP: período médio de postura; NMO: número médio de ovos; MMO: massa média de ovos; PMI: período médio de incubação; PMEL: percentual médio de eclosão larval; RE: reprodução estimada; IN: índice nutricional; NMP: número médio de postura.

controladas $\left(30^{\circ} \mathrm{C}\right.$ e $75 \%$ de UR) e $10,7 \pm 3,0$ dias em condições naturais na cidade de Cairo, Egito. HAFEZ et al. (1972) estudaram o efeito da temperatura e umidade sobre os aspectos biológicos de $\boldsymbol{A}$. $(\boldsymbol{P}$.) arboreus, demonstrando que em temperaturas baixas $\left(20^{\circ} \mathrm{C}\right)$ os períodos de pré-postura e postura são acentuadamente prolongados.

Número e ritmo de posturas

As fêmeas realizaram de 1 a 18 posturas $(13,48 \pm 5,74$ posturas $)$ em B.O.D, de 3 a $9(5,41 \pm 2,65)$ na ECH e 3 a 12 posturas $(8,15 \pm 3,16$ posturas) na ES. SCHUMAKER \& OBA (1988) observaram um número de posturas $(\mathrm{n}=11)$ próximo do obtido no presente estudo. ROHR (1909) verificou até seis posturas para $\boldsymbol{A}$.(P.) miniatus em condições naturais. Nas ES e ECH o número de postura foi significativamente menor, provavelmente devido ao estresse climático, fazendo com que a fêmea direcione sua energia para processos metabólicos destinados à sobrevivência e não à reprodução, diminuindo, assim, o número de posturas.

O ritmo de postura das fêmeas nas três condições evidencia pico de postura no terceiro dia
(B.O.D e ECH) e no quarto dia (ES). Nas três condições estudadas, o ritmo de postura apresentou o mesmo comportamento: poucos ovos no início da postura, atingindo um número máximo de ovos entre o terceiro e quarto dias e queda progressiva até o final da postura (Figura 1a).

Número de ovos

O número de ovos produzido entre todas as posturas realizadas variou entre 46 e 138 ovos para as fêmeas ( $\mathrm{n}=22)$ mantidas em B.O.D, 41 e 108 ovos para as fềmeas da $\mathrm{ECH}(\mathrm{n}=22)$ e 74 e 138 ovos para as fêmeas da $\mathrm{ES}(\mathrm{n}=22)$. Ao avaliar o número médio de ovos, verificou-se que as fêmeas mantidas em B.O.D produziram $100,14 \pm 27,82$ ovos por postura e 1350 ovos em todas as posturas, enquanto as fêmeas mantidas na ECH e ES ovipositaram 81,95 20,18 e 109,70 $\pm 21,12$ ovos por postura, e 443 e 894 ovos em todas as posturas, respectivamente. Tal variação pode ser consequência do retardo da maturidade do órgão reprodutor, causado por fatores climáticos adversos (temperatura e/ou umidade), sofridos nos estágios anteriores, visto que não houve diferença significativa $(\mathrm{P}>0,05)$ na quantidade de sangue ingerida pelas fêmeas 


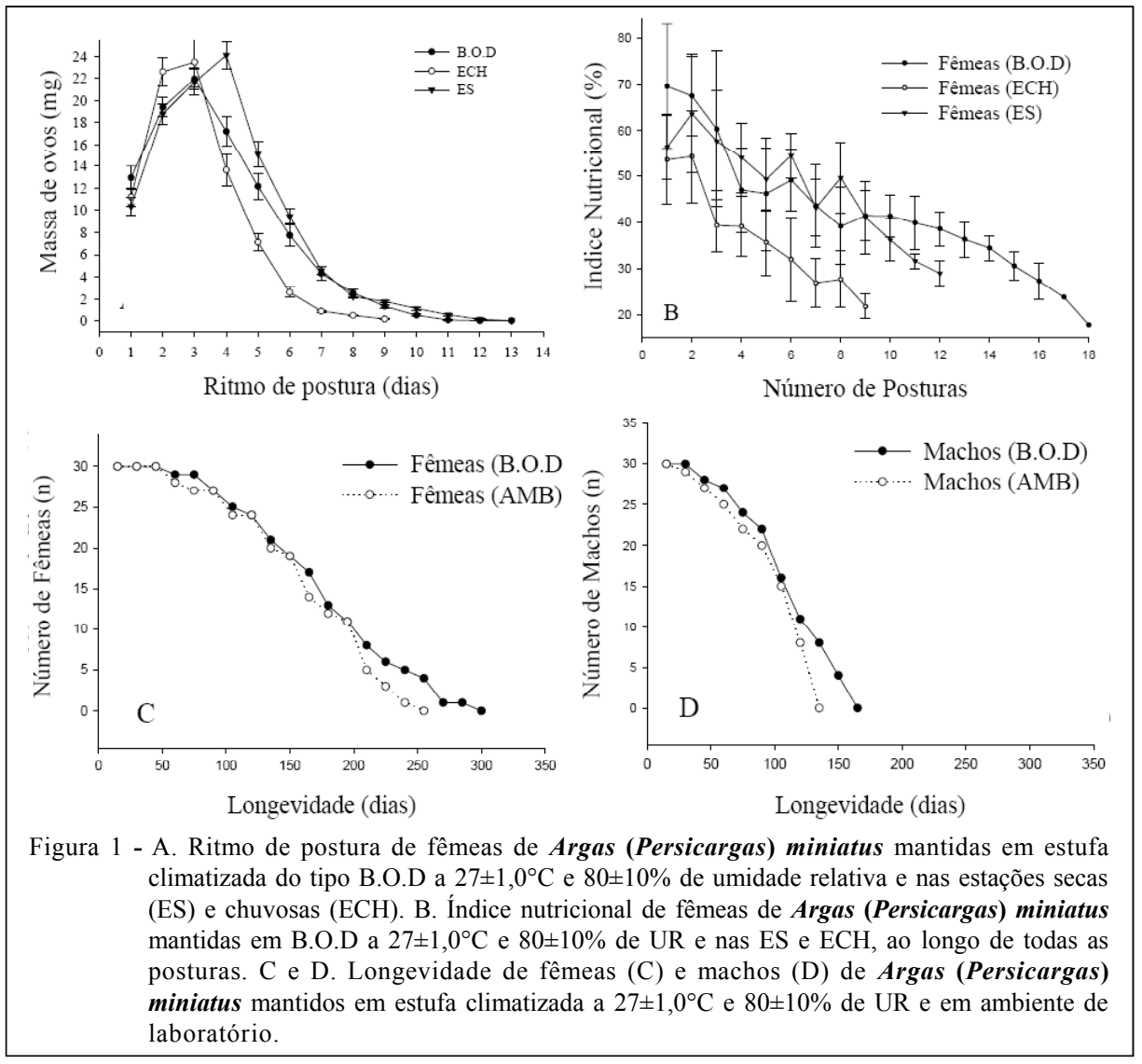

nas três condições experimentais. Nas ECH, as fêmeas tiveram menor $(\mathrm{P}<0,05)$ capacidade de produzir ovos.

O número médio de ovos da primeira postura $(79,82 \pm 23,54$ e $93,23 \pm 10,75$ ovos) foi significativamente inferior aos da segunda $(90,42 \pm 24,15$ e $115,23 \pm 13,97$ ovos) e terceira $(96,16 \pm 20,24$ e $125,73 \pm 11,88$ ovos) posturas $(\mathrm{P}<0,05)$ durante as ECH e ES, respectivamente. Segundo HOOGSTRAAL et al. (1975), o número de ovos nas duas primeiras posturas de $\boldsymbol{A}$. (P.) robertsi é menor porque as fêmeas consomem parte do sangue ingerido para completar o desenvolvimento gonadal e para a manutenção metabólica da fêmea jovem. No entanto, para as fêmeas mantidas em B.O.D, o número de ovos não diferiu nos primeiros dias de postura, com $120,63 \pm 36,65$ ovos na primeira postura, $135,62 \pm 30,41$ ovos na segunda e $137,88 \pm 43,23$ ovos na terceira, provavelmente porque as fêmeas de $\boldsymbol{A}$. $(\boldsymbol{P}$.) miniatus atingiram maturidade sexual precocemente e a energia produzida a partir do sangue ingerido foi destinada à produção de ovos e não para completar o desenvolvimento do aparelho reprodutivo.

Índice nutricional

$\mathrm{O}$ índice nutricional das fêmeas mantidas em B.O.D $(41,86 \pm 6,5)$, na ECH $(36,68 \pm 6,8)$ e na ES
$(47,17 \pm 6,73)$, para todas as posturas, variou significativamente $(\mathrm{P}<0,05)$ (Tabela 1). A eficiência com que as fêmeas converteram a energia em ovos, a partir do sangue ingerido, diminuiu a partir da terceira postura em B.O.D e na ECH e na quarta postura para as fêmeas mantidas na ES. Isso refletiu em uma menor quantidade de ovos ao longo das posturas, em todas as condições experimentais estudadas (Figura 1b).

Estudos conduzidos com carrapatos ixodídeos indicam uma correlação significativa entre o peso da fêmea e o número de ovos (DRUMMOND et al., 1969). Os resultados obtidos no presente estudo demonstram que essa correlação é de aproximadamente $75 \%(\mathrm{P}<0,05)$ até a quarta postura, para as fêmeas mantidas em B.O.D, e até a quinta e oitava posturas, para as fêmeas durante as ECH e ES, respectivamente. A partir dessas posturas, essa correlação diminui progressivamente. Contudo, quando a correlação entre massa de ovos e o peso da fêmea no final da postura foi calculada a cada postura, verificou-se que essa correspondência se mantinha acima de $70 \%(\mathrm{P}<0,05)$ em todas as posturas das fêmeas mantidas nas três condições experimentais. 
Percentual de eclosão

O percentual médio de eclosão das larvas em B.O.D foi de 90,59 $\pm 6,53 \%$, significativamente superior $(\mathrm{P}<0,05)$ ao obtido nas $\mathrm{ECH}(85,57 \pm 6,42 \%)$ e ES $(86,08 \pm 5,9 \%)$. O menor percentual de eclosão das larvas durante a estação chuvosa pode ser atribuído à elevada temperatura desse período.

Peso médio dos ovos

O peso médio dos 10 grupos de 100 ovos foi de $0,01368 \pm 0,00048 \mathrm{~g}$. Em relação à quantidade de ovos por grama de postura, pode-se dizer que um ovo $\operatorname{de} \boldsymbol{A}$. (P.) miniatus pesa $0,14 \pm 0,0048 \mathrm{mg}$, logo, um grama de ovo contém $7317,86 \pm 253,09$ ovos.

\section{Longevidade}

O período médio de longevidade para adultos mantidos em B.O.D e em ambiente de laboratório foi $116,50 \pm 49,75$ dias e $103 \pm 41,08$ dias para machos e $180,50 \pm 88,74$ dias e $167,50 \pm 75,75$ dias para fêmeas. $O$ ritmo de mortalidade foi mais acentuado para os adultos mantidos em condição ambiente de laboratório (Figura 1c). Os machos sobreviveram por um período menor $(\mathrm{P}<0,05)$ do que as fêmeas em todas as condições avaliadas, diferindo dos resultados encontrados em $\boldsymbol{A}$. (P.) arboreus (HAFEZ et al., 1972), que não apresentaram diferença na longevidade de machos e fêmeas mantidos sob mesma temperatura e umidade. KAISER (1965) verificou para $\boldsymbol{A}$. (P.) arboreus mantidos sob condições controladas um período de longevidade máximo de 19 meses, enquanto que HAFEZ et al. (1972) verificaram que existe uma relação inversa entre temperatura e longevidade de $\boldsymbol{A}$. (P.) arboreus: três anos a $21^{\circ} \mathrm{C}$ e 3 meses a $40^{\circ} \mathrm{C}$ e que, em ambientes com baixo teor de umidade, a longevidade tende a diminuir.

\section{CONCLUSÃO}

Um grama de ovo de $\boldsymbol{A}$. (P.) miniatus contém aproximadamente 7317 ovos e o número de posturas, assim como a quantidade de ovos por postura, varia conforme a condição a que a fêmea é submetida. Há maior sobrevivência de $\boldsymbol{A}$. (P.) miniatus mantidos em B.O.D a $27 \pm 1^{\circ} \mathrm{C}$ e $80 \pm 10 \%$ UR quando comparado à condição ambiental com temperatura e umidade relativa não controlada, porém a longevidade de fêmeas de $\boldsymbol{A}$. (P.) miniatus é maior que a de machos. Os machos de A. (P.) miniatus são originados de ninfas que apresentam menor peso, enquanto que as fêmeas originam-se de ninfas de maior peso. A razão sexual é dependente do instar de origem, com predominância de machos originados de N2 e fêmeas de N3. De um modo geral, os parâmetros biológicos do estágio adulto de $\boldsymbol{A}$. (P.) miniatus sofrem alterações negativas quando mantidos em condições não controladas, ocorrendo com maior intensidade durante a ECH do ano.

\section{REFERÊNCIAS}

BENNETT, G.F. Ovoposition of Boophilus microplus (CANESTRINI) (ACARIDA: IXODIDAE) I. Influence of tick size on egg production. Acarologia, v.16, n.1, p.52-61, 1974. Disponível em: < http://lrs.afpmb.org/U0AFQBQ5VADR3NZ4AU7EGUBX/ arc/al_06 _tit_fetch/1/82512>. Acesso em: 17 maio, 2010.

DRUMMOND, R.O. et al. Biology and colonization of the winter tick in the laboratory. Journal of Economic Entomology, v.52, n.1, p.235-238, 1969. Disponível em: <http://lrs.af pmb .org /U0AFQBQ5VADR3NZ4AU7EGUBX/arc/al_06_tit_fetch/ 1/46268>. Acesso em: 17 maio, 2010.

DRUMMOND, R.O. et al. Laboratory testing of insecticides for control of the winter tick. Journal Economic Entomology, v.64, n.1, p.686-688, 1971. Disponível em: <http://lrs.afpmb.org/ U0AFQBQ5VADR3NZ4AU7EGUBX/arc/al_06_tit_fetch/1/ 59828>. Acesso em: 17 maio, 2010.

EL KAMMAH, K.M.; WAHAB, K.S.A. Argas (Persicargas) persicus life cycle under controlled and outdoor conditions. Acarologia, v.21, n.2, p.112-119, 1979. Disponível em: $<$ http://lrs.afpmb.org/UKN7S9V9SWS8UPCYWH1858ZN/arc/ al_06_tit_fetch/2/107927> Acesso em: 17 maio, 2010.

HAFEZ, M. et al. The subgenus Persicargas (Ixodoidea, Argasidae, Argas). 14. Biological studies on the adult stage of A. (P.) arboreus Kaiser, Hoogstraal \& Kohls in Egypt. Journal of Medical Entomology, v.9, n.1, p.19-29, 1972. Disponível em: <http://www.ncbi.nlm. nih.gov/pubmed/5019203>. Acesso em: 17 maio, 2010.

HOOGSTRAAL, H. et al. The subgenus Persicargas (Ixodoidea, Argasidae, Argas). 27. The life cycle of $\boldsymbol{A}$. (P.) robertsi population samples from Taiwan, Thailand, Indonesia, Australia and Sri Lanka. Journal of Tropical Medicine and Public Health, v.6, n.4, p. 18-26, 1975. Dispnível em: <http://lrs.afpmb.org/ U0AFQBQ5VADR3NZ4AU7EGUBX/arc/al_06_tit_fe tch/1/ 186597>. Acesso em: 17 maio, 2010.

KAISER, M.N. The subgenus Persicargas (Ixodoidea, Argasidae, Argas). 3. The life cycle of $\boldsymbol{A}$. (P.) arboreus, and a standardized rearing method for Argasid ticks. Annals of the Entomological Society of America, v.59, n.3, p.496-502, 1965. Disponível em: <http://www.ingentaconnect.com/ content/esa/aesa/1966/00000059/00000003/art00013>. Acesso em: 18 maio, 2010.

KHALIL, G.M. The subgenus Persicargas (Ixodoidea, Argasidae, Argas). 31. The life cycle of $\boldsymbol{A}$. (P.) persicus in the laboratory. Journal of Medical Entomology, v.16, n.3, p.200-206, 1979. Disponível em: <http://www.ingentaconnect.com/content/esa/ jme/1979/00000016/00 000003/art00004>. Acesso em: 18 maio, 2010 .

LABRUNA, M.B. et al. Study of weight of eggs from six ixodid species from Brazil. Memórias do Instituto Oswaldo Cruz, v.92, n.2, p. 205-207, 1997. Disponível em: <http:// 
memorias.ioc.fiocruz.br/922/3193.html>. Acesso em: 17 maio, 2010 .

MAGALHÃES, F.E.P. Novos aspectos morfológicos, biológicos e tóxicos de Argas (Persicargas) miniatus Koch, 1844 (Ixodoidea, Argasidae) no Estado do Rio de Janeiro. 1979. 95f. Dissertação (Mestrado em Parasitologia Veterinária) - Universidade Federal Rural do Rio de Janeiro, Seropédica, RJ.

MAGALHÃES, F.E.P. et al. Paralysis in Gallus gallus and Carina moschata induced by larvae of Argas (Persicargas) miniatus. Pesquisa Veterinária Brasileira,v.7. n.2, p.47-49, 1987. Disponível em: <http://1rs.afpmb.org/ U0AFQBQ5VADR3NZ4AU7EGUBX/arc/al_06_tit_fetch/ 1/139470>. Acesso em: 17 maio, 2010.

MARCHOUX, E.; SALIMBENI, A. La spirillose des poules. Annales de I'Institut Pasteur Lille, Paris, v.17, n.1, p.569-580, 1903.
ROHR, C.J. Estudo sobre Ixodidas do Brasil. Rio de Janeiro: Gomes, Irmão \& C., 1909. 226p.

SANTOS, H.A. et al. Aspectos biológicos de larvas de Argas (Persicargas) miniatus Koch, 1844, (Ixodoidea: Argasidae) em Gallus gallus. Revista Brasileira de Parasitologia Veterinária, v.17, supl.1, p.45-49, 2008. Disponível em: $<$ http://www.cbpv.com.br/rbpv /documentos/17supl.12008/ Artropode009.pdf $>$. Acesso em: 17 maio, 2010.

SCHUMAKER, T.T.S.; OBA, M.S.P. Aspectos Morfo-biológicos de Argas (Persicargas) miniatus. Koch, 1844 (Ixodoidea, Argasidae). Revista Brasileira de Entomologia, v.32, n.2, p.161-173, 1988 .

SONENSHINE, D.E. Biology of ticks. New York: Oxford University, 1993. V.2, 447p. 